\title{
Identification of intrinsic long-range degree correlations in complex networks
}

\author{
Yuka Fujik* and Kousuke Yakubd \\ Department of Applied Physics, Hokkaido University, Sapporo 060-8628, Japan
}

(Dated: January 31, 2020)

\begin{abstract}
Many real-world networks exhibit degree-degree correlations between nodes separated by more than one step. Such long-range degree correlations (LRDCs) can be fully described by one joint and four conditional probability distributions with respect to degrees of two randomly chosen nodes and shortest path distance between them. While LRDCs are induced by nearest-neighbor degree correlations (NNDCs) between adjacent nodes, some networks possess intrinsic LRDCs which cannot be generated by NNDCs. Here we develop a method to extract intrinsic LRDC in a correlated network by comparing the probability distributions for the given network with those for nearestneighbor correlated random networks. We also demonstrate the utility of our method by applying it to several real-world networks.
\end{abstract}

PACS numbers: 89.75.Hc, 89.75.Fb, 02.70.Rr

\section{INTRODUCTION}

A network representation is one of the most general and efficient ways to describe systems consisting of elements interacting with each other [1 $[3]$. In many networks for real-world complex systems, the number of edges from a node, namely degree, widely fluctuates from node to node and often obeys a power-law distribution [4]. Such broad distributions of degrees may bring about distinctive complexity into networks, that is, correlations between degrees of two nodes [5]. Previous works on degree correlations have focused mainly on nearest neighbor degree correlations (NNDCs) between adjacent nodes, because NNDCs in complex networks influence their structural features [6, 7] and dynamical properties [8, 9]. In recent years, however, it has been elucidated that long-range degree correlations (LRDCs) beyond nearest neighbor nodes are deeply related to various properties of networks. In fractal complex networks like the World Wide Web, for example, highly connected (hub) nodes repel each other over long shortest path distances [10]. Distances between hub nodes are known to play an important role also in the spreading of congestion 11], the existence of an epidemic threshold [12], and other properties of functional networks [13, 14]. These properties cannot be explained by NNDC. For quantifying such long-range correlations systematically, a framework to analyze LRDCs in complex networks has recently been formulated by introducing several probability distributions describing LRDC [15]. By comparing the probability distributions for a given network with those for the corresponding random networks with the same degree distribution, one can prove the existence of LRDC in the network and obtain detailed information on the LRDC.

It should be emphasized that NNDC generally induces LRDC. Let us consider, for example, a network in which

\footnotetext{
* y-fujiki@eng.hokudai.ac.jp

† yakubo@eng.hokudai.ac.jp
}

nodes are randomly connected under the condition that high degree nodes are preferably connected to low degree nodes (disassortative mixing), as shown in Fig. 1. In this network, two nodes with similar degrees are always separated by an even number of the shortest path distance $l$ as if antiferromagnetic spins lying in next nearest neighbor show the ferromagnetic order. This implies that the network shows long-range assortative mixing at $l=2,4, \cdots$. Such an extrinsic LRDC induced by NNDC can also be understood from the fact that maximally assortative or disassortative networks display community structures [6]. It is impossible to judge whether observed properties of a correlated network are caused by NNDC or intrinsic LRDC that cannot be produced by NNDC, if intrinsic LRDC is not segregated from extrinsic one. In order to extract the influence of intrinsic LRDCs on structural or dynamical properties of networks separately from the influence of NNDCs, the identification of intrinsic LRDCs is essential. This enables us to investigate the role of LRDCs in various phenomena on complex networks, such as percolation, synchronization, and epidemic spread. In the previous framework [15], however, we cannot distinguish intrinsic LRDCs from extrinsic ones. We provide, in this paper, a method to discriminate between extrinsic and intrinsic LRDCs in a correlated network. To this end, we calculate the probability distributions describing LRDCs in nearest-neighbor correlated random networks (NNCRNs) which are maximally randomized networks under the constraint that NNDC is specified. One can identify intrinsic LRDC in a network by comparing the

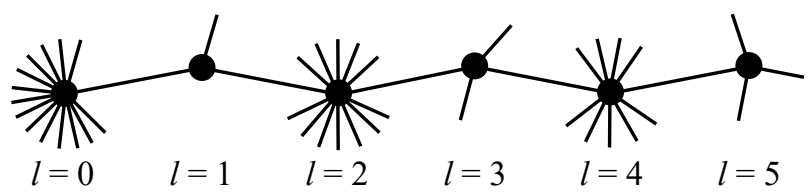

FIG. 1. Schematic view of a node chain in a disassortative network. Two nodes separated by an even (odd) distance have similar (dissimilar) degrees. 
probability distributions with those for its corresponding NNCRNs having the same NNDC as the original network. We also demonstrate the utility of our method by applying it to several real-world networks.

The rest of this paper is organized as follows. In Sec. [I] we briefly summarize the previous work [15] regarding the general description of LRDCs. Examples of extrinsic LRDCs induced by NNDCs are clearly shown by means of the probability distributions. In Sec. [III we present the mean-field calculation of the probability distributions for NNCRNs in order to extract intrinsic LRDCs. We demonstrate, in Sec. IV how to interpret the observed LRDCs by applying our method to several real-world networks. Finally, we conclude in Sec. V]

\section{IDENTIFICATION OF LONG-RANGE DEGREE CORRELATIONS}

In this section, we briefly explain the way to describe LRDCs and to judge the existence of them in complex networks, according to the previous work 15]. We also demonstrate that NNDC inevitably induces LRDC even in a network with no intrinsic LRDCs.

\section{A. Description of LRDC}

A general pairwise LRDC can be described by the joint probability distribution $P\left(k, k^{\prime}, l\right)$ that two randomly chosen nodes have degrees $k$ and $k^{\prime}$ and the shortest path distance between them is $l$. Let us assume that $P\left(k, k^{\prime}, l\right)$ is defined also for pairs of identical nodes $(l=0)$ and for disconnected node pairs. Thus, $P\left(k, k^{\prime}, l\right)$ is normalized as $\sum_{k, k^{\prime}, l} P\left(k, k^{\prime}, l\right)=1$, where the summation over $l$ includes $l=0$ and the virtual distance (denoted by $l_{\infty}$ ) between disconnected pairs. If the distribution $\tilde{P}\left(k, k^{\prime}, l\right)$ defined only for connected pairs is required, one can construct it from $P\left(k, k^{\prime}, l\right)$ as $\tilde{P}\left(k, k^{\prime}, l\right)=P\left(k, k^{\prime}, l\right) / \sum_{k, k^{\prime}} \sum_{l=1}^{\prime} P\left(k, k^{\prime}, l\right)$, where $\sum_{l}^{\prime}$ does not include $l=l_{\infty}$. In addition to the joint probability, it is convenient to introduce four conditional probability distributions $P\left(l \mid k, k^{\prime}\right), P\left(k^{\prime} \mid k, l\right), P\left(k, k^{\prime} \mid l\right)$, and $P\left(k^{\prime}, l \mid k\right)$ which are defined by

$$
\begin{aligned}
& P\left(l \mid k, k^{\prime}\right)=\frac{P\left(k, k^{\prime}, l\right)}{\sum_{l} P\left(k, k^{\prime}, l\right)}, \\
& P\left(k^{\prime} \mid k, l\right)=\frac{P\left(k, k^{\prime}, l\right)}{\sum_{k^{\prime}} P\left(k, k^{\prime}, l\right)}, \\
& P\left(k, k^{\prime} \mid l\right)=\frac{P\left(k, k^{\prime}, l\right)}{\sum_{k, k^{\prime}} P\left(k, k^{\prime}, l\right)}, \\
& P\left(k^{\prime}, l \mid k\right)=\frac{P\left(k, k^{\prime}, l\right)}{\sum_{k^{\prime}, l} P\left(k, k^{\prime}, l\right)} .
\end{aligned}
$$

The meanings of these probability distributions are obvious. The probability $P\left(l \mid k, k^{\prime}\right)$, for example, is the shortest path distance distribution between nodes of degrees $k$ and $k^{\prime}, P\left(k^{\prime} \mid k, l\right)$ is the degree distribution of a node separated by $l$ from a node of degree $k$, and so on. LRDC in a network can be characterized by one of these five probability distributions. We should note that $P\left(k, k^{\prime}, l\right), P\left(l \mid k, k^{\prime}\right)$, and $P\left(k^{\prime}, l \mid k\right)$ are meaningless for networks with infinitely large components, because these distributions are always zero for finite $l$.

The above probability distributions also describe degree correlation between nearest-neighbor nodes. It is well understood that NNDC is captured by the joint probability $P_{\mathrm{nn}}\left(k, k^{\prime}\right)$ that two end nodes of a randomly chosen edge have the degrees $k$ and $k^{\prime}$ or the conditional probability $P_{\mathrm{nn}}\left(k^{\prime} \mid k\right)$ that a node adjacent to a randomly chosen node of degree $k$ has the degree $k^{\prime}$ [16, 17]. These probability distributions $P_{\mathrm{nn}}\left(k, k^{\prime}\right)$ and $P_{\text {nn }}\left(k^{\prime} \mid k\right)$ are equivalent to $P\left(k, k^{\prime} \mid l=1\right)$ and $P\left(k^{\prime} \mid k, l=\right.$ $1)$, respectively. In this context, the probability distributions describing LRDC are a natural extension of $P_{\mathrm{nn}}\left(k, k^{\prime}\right)$ and $P_{\mathrm{nn}}\left(k^{\prime} \mid k\right)$. In fact, the well-known relation $P_{\mathrm{nn}}\left(k^{\prime} \mid k\right)=P_{\mathrm{nn}}\left(k, k^{\prime}\right) / \sum_{k^{\prime}} P_{\mathrm{nn}}\left(k, k^{\prime}\right)$ is a special case of $P\left(k^{\prime} \mid k, l\right)=P\left(k, k^{\prime} \mid l\right) / \sum_{k^{\prime}} P\left(k, k^{\prime} \mid l\right)$.

Using the joint and conditional probability distributions, we can define various useful indices reflecting specific aspects of LRDC. For example, the average degree of the $l$ th neighbor nodes of a node of degree $k$ is defined by

$$
k_{l}(k)=\sum_{k^{\prime}} k^{\prime} P\left(k^{\prime} \mid k, l\right),
$$

which is an extension of the average degree of neighbors of a node of degree $k, k_{\mathrm{nn}}(k)=\sum_{k^{\prime}} k^{\prime} P_{\mathrm{nn}}\left(k^{\prime} \mid k\right)$ [18]. Another example is the average shortest path distance between two nodes of degrees $k$ and $k^{\prime}$, which is defined by

$$
\left\langle l\left(k, k^{\prime}\right)\right\rangle=\sum_{l} l \tilde{P}\left(l \mid k, k^{\prime}\right)
$$

where $\tilde{P}\left(l \mid k, k^{\prime}\right)=P\left(l \mid k, k^{\prime}\right) / \sum_{l=1}^{\prime} P\left(l \mid k, k^{\prime}\right)$ is the distribution defined only for connected pairs. By means of this index, we can discuss long-range repulsive or attractive correlation between hub nodes. Besides the above, many indices characterizing NNDCs, such as assortativity [5], can be extended for LRDCs [19, 20].

\section{B. Existence of LRDC}

A network has LRDC if the probability distributions for the network are different from those for a long-range uncorrelated network with the same degree distribution. In the case of nearest-neighbor uncorrelated networks, the joint probability satisfies $P_{\mathrm{nn}}\left(k, k^{\prime}\right)=Q_{\mathrm{nn}}(k) Q_{\mathrm{nn}}\left(k^{\prime}\right)$, where $Q_{\mathrm{nn}}(k)=k P(k) /\langle k\rangle$ is the probability that one end node of a randomly chosen edge has the degree $k$, $P(k)$ is the degree distribution, and $\langle k\rangle$ is the average degree. Analogously, a long-range uncorrelated network 
is considered to be defined as a network for which the relation

$$
P\left(k, k^{\prime} \mid l\right)=Q(k \mid l) Q\left(k^{\prime} \mid l\right),
$$

is satisfied for any $l$, where $Q(k \mid l)=\sum_{k^{\prime}} P\left(k, k^{\prime} \mid l\right)$ is the probability that one node of a randomly chosen node pair separated by $l$ from each other has the degree $k$. The condition of Eq. (4) can be established if the network size is infinite, while $P\left(k, k^{\prime}, l\right), P\left(l \mid k, k^{\prime}\right)$, and $P\left(k^{\prime}, l \mid k\right)$ are meaningless in such networks. In finite networks, in contrast, the finite-size effect prevents to hold Eq. (4) even in random networks. It is, however, rather practically important to compare LRDC in a given network with that in a random network with the same size and the same degree distribution. Hereafter, a network $G$ is regarded to have LRDC if the probability distributions for $G$ differ from those for the corresponding random network. We denote the probability distribution functions for random networks by adding the subscript 0 .

One can analytically calculate the five probability distributions for a random network with a given size $N$ and a given degree distribution $P(k)$ within the mean-field approximation [15, 21]. In this theory, the shortest path distance distribution $P_{0}\left(l \mid k, k^{\prime}\right)$ between nodes of degrees $k$ and $k^{\prime}$ is first computed, then the joint probability distribution $P_{0}\left(k, k^{\prime}, l\right)$ is calculated from the general relation $P_{0}\left(k, k^{\prime}, l\right)=P(k) P\left(k^{\prime}\right) P_{0}\left(l \mid k, k^{\prime}\right)$. Furthermore, Eq. (11) presents the other three conditional probability distributions. Details of the calculations will be explained in Sec. III The accuracy of the above theoretical calculation is basically quite high, while it becomes relatively poor if the random network is very close to the percolation transition because the mean-field theory assumes a narrow distribution of component sizes. The existence of LRDC in a network $G$ can be proven by comparing $P\left(k, k^{\prime}, l\right)$ (or other distributions) for $G$ with $P_{0}\left(k, k^{\prime}, l\right)$ calculated theoretically for the corresponding random network with the same $N$ and the same $P(k)$ as $G$.

\section{Extrinsic LRDC}

Within the above framework, it has been revealed that many real-world networks exhibit LRDCs 15. As mentioned in Sec. I] observed degree correlations, however, include extrinsic LRDCs which are purely generated by NNDCs. Here we demonstrate numerically how NNDCs induce LRDCs. For this purpose, we prepare nearest-neighbor correlated random networks (NNCRNs) in which nodes are randomly connected while a specific NNDC is preserved. More specifically, we first rewire, referring to the procedure adopted in [6], edges in an Erdős-Rényi random graph to increase or decrease the Spearman's rank correlation coefficient $\varrho$ defined by [22]

$$
\varrho=\frac{\sum_{k, k^{\prime}} R_{k} R_{k^{\prime}} P_{\mathrm{nn}}\left(k, k^{\prime}\right)-\left[\sum_{k} R_{k} Q_{\mathrm{nn}}(k)\right]^{2}}{\sum_{k} R_{k}^{2} Q_{\mathrm{nn}}(k)-\left[\sum_{k} R_{k} Q_{\mathrm{nn}}(k)\right]^{2}},
$$

where $R_{k}$ given by [23]

$$
R_{k}=\frac{N k P(k)+1}{2}+N \sum_{k^{\prime}=0}^{k-1} k^{\prime} P\left(k^{\prime}\right),
$$

is the rank of the degree $k$. The correlation coefficient $\varrho$ has a similar meaning to assortativity $r[5]$, but does work well even for heterogeneous networks with the diverging third-order moment of the degree distribution, unlike the case of $r$. When $\varrho$ becomes a desired value, the assortative/disassortative rewiring operation is switched to a random rewiring procedure while keeping $P_{\mathrm{nn}}\left(k, k^{\prime}\right)$. After a sufficient number of random rewiring operations, we have an assortative/disassortative NNCRN with the same degree distribution as the initial Erdős-Rényi random graph.

Figure 2 represents the probability distribution $P\left(k, k^{\prime} \mid l\right)$ measured for NNCRNs which are numerically prepared by the above method. The network size is $N=1,000$ and the average degree is set as $\langle k\rangle=5.0$. The distribution $P\left(k, k^{\prime} \mid l\right)$ is averaged over 100 samples. The upper five panels show the results for the assortative NNCRN with $\varrho=0.8$ as functions of $k$ and $k^{\prime}$ for $l=1$ to 5 (from left to right). The distribution $P\left(k, k^{\prime} \mid l=1\right)$ clearly indicates a strong positive correlation between $k$ and $k^{\prime}$. This tendency is found up to about $l=3$ but becomes weaker as $l$ increases. For $l=5$, in contrast, $P\left(k, k^{\prime} \mid l\right)$ displays weak negative degree correlation caused by the reaction of assortative mixing for $l \leq 3$. The results for the disassortative NNCRN with $\varrho=-0.8$ are shown in the lower panels. These results demonstrate that the sign of the correlation between $k$ and $k^{\prime}$ alternately changes as $l$ is incremented. This behavior of $P\left(k, k^{\prime} \mid l\right)$ can be easily understood from Fig. 1. Such an alternative correlation-sign change in nearestneighbor correlated orders can also be seen in an antiferromagnetic spin ordering and a color ordering of graph coloring for tree-like graphs. Assortative and disassortative degree correlations shown in Fig. 2 for $l \geq 2$ are purely induced by NNDCs, namely, extrinsic LRDCs. The purpose of this work is to distinguish between extrinsic and intrinsic LRDCs in a network with both types of correlations.

\section{MEAN-FIELD ANALYSIS}

As LRDC in a given network is detected by comparing its probability distributions with those for the corresponding random network, we can identify intrinsic LRDC by comparing with those for the corresponding NNCRN with the same size and the same NNDC. In this section, we analytically calculate the probability distributions for an NNCRN with a given size $N$ and a given $P_{\mathrm{nn}}\left(k, k^{\prime}\right)$ within the mean-field approximation and the local-tree approximation. Hereafter, we denote the probability distribution functions for NNCRNs by adding the subscript 1. 


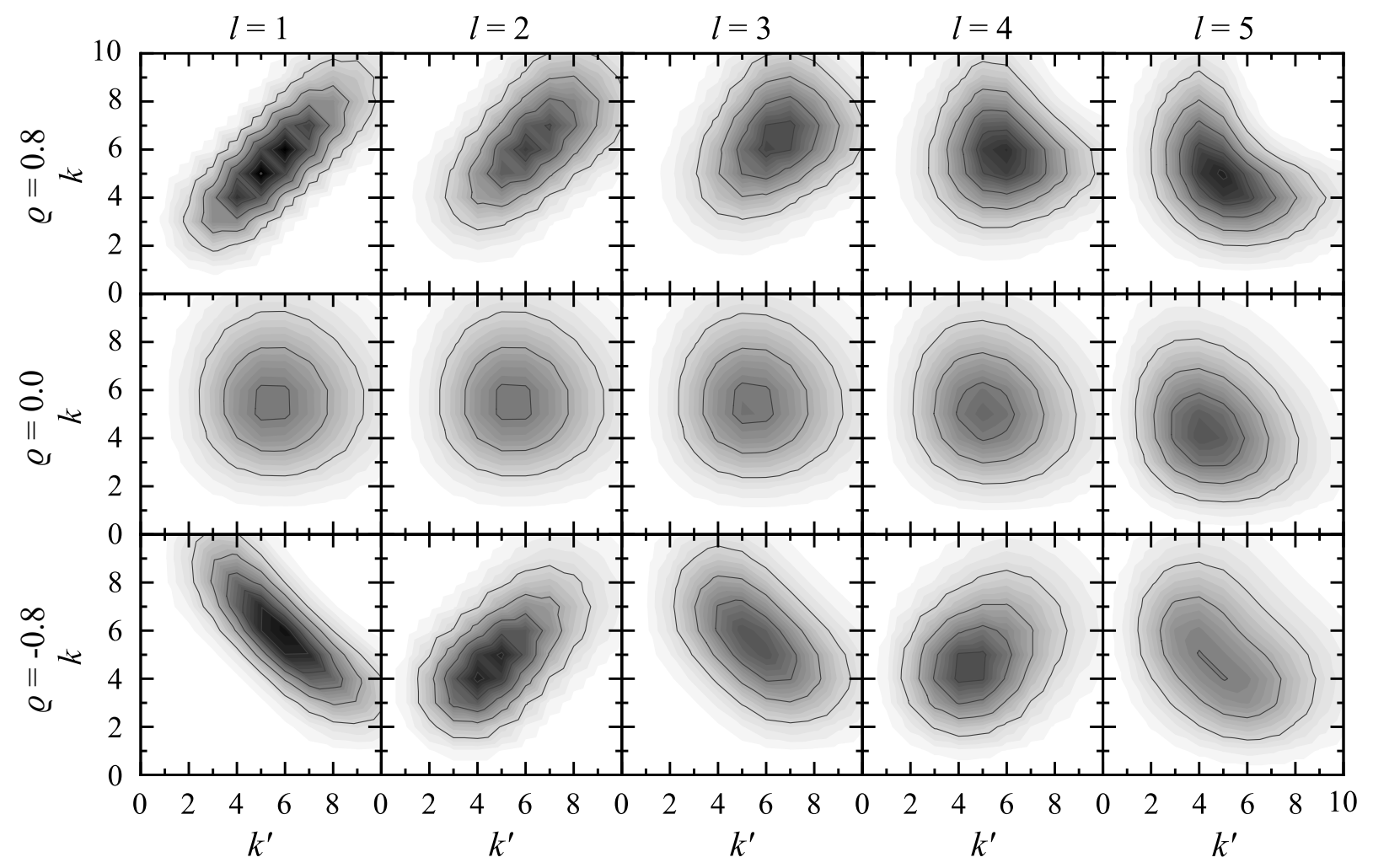

FIG. 2. Contour plots of the probability distribution $P\left(k, k^{\prime} \mid l\right)$ for NNCRNs with $\varrho=0.8$ (upper panels, $r=0.76$ ), 0.0 (middle panels, $r=0.0$ ), and -0.8 (lower panels, $r=-0.75$ ) as functions of $k$ and $k^{\prime}$ for $l=1$ to 5 , where $r$ is the assortativity [5]. The degree sequence is the same as that of Erdös-Rényi random graphs with $N=1,000$ and $\langle k\rangle=5.0$.

We first focus on the shortest path distance distribution $P_{1}\left(l \mid k, k^{\prime}\right)$ between nodes of degrees $k$ and $k^{\prime}$. Such a distribution for a connected NNCRN has been calculated by Melnik and Gleeson [21]. The distribution $P_{1}\left(l \mid k, k^{\prime}\right)$ for a network with multiple components can be calculated by extending their argument. As mentioned in Sec. IA $P_{1}\left(l \mid k, k^{\prime}\right)$ is normalized with respect to $l$ including $l=0$ and the virtual distance $\left(l_{\infty}\right)$ between disconnected pairs. In order to calculate $P_{1}\left(l \mid k, k^{\prime}\right)$ both for connected and disconnected node pairs, let us introduce the cumulative probability $\rho\left(l \mid k, k^{\prime}\right)=\sum_{l^{\prime}=0}^{l} P_{1}\left(l^{\prime} \mid k, k^{\prime}\right)$ only for $l \neq l_{\infty}$. This probability is expressed by

$$
\rho\left(l \mid k, k^{\prime}\right)=1-\left[1-\frac{\delta_{k k^{\prime}}}{N P(k)}\right]\left[1-\bar{q}\left(l-1 \mid k, k^{\prime}\right)\right]^{k},
$$

where $\bar{q}\left(l \mid k, k^{\prime}\right)$ is the probability that an adjacent node of a randomly chosen node $i_{k}$ of degree $k$ lies within the distance $l$ from a specific node $j_{k^{\prime}}$ of degree $k^{\prime}$, and $\bar{q}\left(-1 \mid k, k^{\prime}\right)=0$. The prefactor $\left[1-\delta_{k k^{\prime}} / N P(k)\right]$ represents the probability that the node $i_{k}$ does not coincide with the node $j_{k^{\prime}}$. Thus, the right-hand side of Eq. (7) is the probability that at least one of $k$ adjacent nodes of $i_{k}\left(\neq j_{k^{\prime}}\right)$ lies within the distance $l-1$ from $j_{k^{\prime}}$. From the definition, the probability $\bar{q}\left(l \mid k, k^{\prime}\right)$ is given by [21]

$$
\bar{q}\left(l \mid k, k^{\prime}\right)=\sum_{k^{\prime \prime}} P_{\mathrm{nn}}\left(k^{\prime \prime} \mid k\right) q\left(l \mid k^{\prime \prime}, k^{\prime}\right)
$$

where $q\left(l \mid k, k^{\prime}\right)$ is the probability that a terminal node $i_{k}$ of a randomly chosen edge lies within the distance $l$ from a node $j_{k^{\prime}}$ of degree $k^{\prime}$ given that $i_{k}$ has the degree $k$ and is closer to $j_{k^{\prime}}$ than the other terminal node of the edge. We notice here that $P(k)$ in Eq. (77) and $P_{\mathrm{nn}}\left(k^{\prime} \mid k\right)$ in Eq. (8) are presented by the specified $P_{\mathrm{nn}}\left(k, k^{\prime}\right)$ as $P(k)=\langle k\rangle \sum_{k^{\prime}} P_{\mathrm{nn}}\left(k, k^{\prime}\right) / k$ and $P_{\mathrm{nn}}\left(k^{\prime} \mid k\right)=\langle k\rangle P_{\mathrm{nn}}\left(k, k^{\prime}\right) / k P(k)$, respectively. A similar idea to Eq. (7) leads to another relation between $\bar{q}\left(l \mid k, k^{\prime}\right)$ and $q\left(l \mid k, k^{\prime}\right)$, which is given by

$$
q\left(l \mid k, k^{\prime}\right)=1-\left[1-\frac{\delta_{k k^{\prime}}}{N P(k)}\right]\left[1-\bar{q}\left(l-1 \mid k, k^{\prime}\right)\right]^{k-1} .
$$

We can iteratively solve the coupled recurrence equations (8) and (9) with the initial condition,

$$
\bar{q}\left(0 \mid k, k^{\prime}\right)=\frac{P_{\mathrm{nn}}\left(k^{\prime} \mid k\right)}{N P\left(k^{\prime}\right)},
$$

which is the probability that an adjacent node of $i_{k}$ is the specific node $j_{k^{\prime}}$ itself. The cumulative probability $\rho\left(l \mid k, k^{\prime}\right)$ is obtained by substituting the solution to Eq. (7).

Although the cumulative probability $\rho\left(l \mid k, k^{\prime}\right)$ must be symmetric with respect to $k$ and $k^{\prime}$ from the definition, $\rho\left(l \mid k, k^{\prime}\right)$ given by Eq. (7) is obviously asymmetric. This 

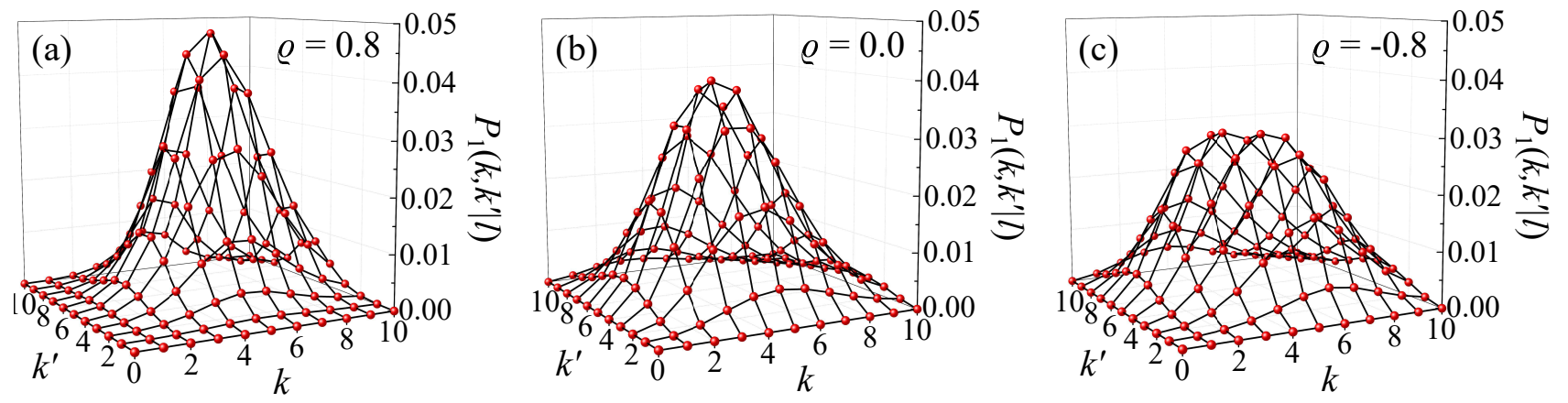

FIG. 3. (Color online) Conditional probability distributions $P_{1}\left(k, k^{\prime} \mid l=5\right)$ as functions of $k$ and $k^{\prime}$ for assortative/disassortative NNCRNs treated in Fig. 2. Red symbols represent the numerical results which are the same as the results for $l=5$ shown in Fig. 22 and the wireframes indicate $P_{1}\left(k, k^{\prime} \mid l=5\right)$ calculated in the mean-field approximation. The Spearman's rank correlation coefficients are (a) $\varrho=0.8$, (b) $\varrho=0.0$, and (c) $\varrho=-0.8$.

is due to the asymmetric treatment of the source node $j_{k^{\prime}}$ and sink node $i_{k}$, and influences the accuracy of the mean-field approximation. For $k^{\prime}>k, \rho\left(l \mid k, k^{\prime}\right)$ is generally more accurate than $\rho\left(l \mid k^{\prime}, k\right)$ [15]. To improve the accuracy, we first calculate $\rho\left(l \mid k, k^{\prime}\right)$ for $k<k^{\prime}$ by Eq. (7), then transfer it to $\rho\left(l \mid k^{\prime}, k\right)$. Using the symmetrized $\rho\left(l \mid k, k^{\prime}\right)$, the probability distribution $P_{1}\left(l \mid k, k^{\prime}\right)$ is then calculated by

$$
P_{1}\left(l \mid k, k^{\prime}\right)=\rho\left(l \mid k, k^{\prime}\right)-\rho\left(l-1 \mid k, k^{\prime}\right),
$$

where $P_{1}\left(0 \mid k, k^{\prime}\right)=\rho\left(0 \mid k, k^{\prime}\right)=\delta_{k k^{\prime}} / N P(k)$, and for disconnected node pairs $P_{1}\left(l_{\infty} \mid k, k^{\prime}\right)=1-\lim _{l \rightarrow \infty} \rho\left(l \mid k, k^{\prime}\right)$. The joint probability distribution $P_{1}\left(k, k^{\prime}, l\right)$ is calculated from the general relation (1a) and $\sum_{l} P\left(k, k^{\prime}, l\right)=$ $P(k) P\left(k^{\prime}\right)$, which is given by

$$
P_{1}\left(k, k^{\prime}, l\right)=P(k) P\left(k^{\prime}\right) P_{1}\left(l \mid k, k^{\prime}\right) .
$$

Other three conditional probability distributions $P_{1}\left(k^{\prime} \mid k, l\right), P_{1}\left(k, k^{\prime} \mid l\right)$, and $P_{1}\left(k^{\prime}, l \mid k\right)$ are computed by Eq. (11).

We have checked the accuracy of the above mean-field approximation. To this end, the same networks as in Fig. 2 have been treated. Figure 3 shows $P\left(k, k^{\prime} \mid l=5\right)$ for (a) $\varrho=0.8$, (b) $\varrho=0.0$, and (c) $\varrho=-0.8$. Black wireframes obtained by the mean-field calculation agree quite well with red symbols indicating the corresponding numerical results. Since the probability distributions other than $P\left(k, k^{\prime} \mid l\right)$ are rigorously related to $P\left(k, k^{\prime} \mid l\right)$ through Eq. (1), the accuracy of the mean-field approximation for these distributions including the joint probability distribution are the same with $P\left(k, k^{\prime} \mid l\right)$.

In order to quantify the accuracy of the mean-field approximation, we introduce the distribution distance defined by

$$
d\left(P, P^{\prime}\right)=\sqrt{\frac{\sum_{k, k^{\prime}} \sum_{l=1}^{\prime}\left[P\left(k, k^{\prime}, l\right)-P^{\prime}\left(k, k^{\prime}, l\right)\right]^{2}}{\sum_{k, k^{\prime}} \sum_{l=1}^{\prime}\left[P\left(k, k^{\prime}, l\right)^{2}+P^{\prime}\left(k, k^{\prime}, l\right)^{2}\right]}},
$$

where $P\left(k, k^{\prime}, l\right)$ and $P^{\prime}\left(k, k^{\prime}, l\right)$ are two joint probability distributions to be compared and the summation $\sum_{l}^{\prime}$ does not include $l=l_{\infty}$. This quantity is bounded as $0 \leq d\left(P, P^{\prime}\right) \leq 1$, where $d\left(P, P^{\prime}\right)=0$ for $P\left(k, k^{\prime}, l\right)=$ $P^{\prime}\left(k, k^{\prime}, l\right)$ and $d\left(P, P^{\prime}\right)=1$ when these two distributions do not overlap each other at all. We have computed the distribution distance $d\left(P_{1}^{\text {ana }}, P_{1}^{\text {num }}\right)$ between two distributions $P_{1}^{\text {ana }}\left(k, k^{\prime}, l\right)$ and $P_{1}^{\text {num }}\left(k, k^{\prime}, l\right)$ calculated analytically and numerically, respectively, for NNCRNs used in Fig. 3. The results are $d\left(P_{1}^{\text {ana }}, P_{1}^{\text {num }}\right)=0.027,0.016$, and 0.013 for $\varrho=0.8,0.0$, and -0.8 , respectively. These distribution distances very closed to zero quantitatively indicate the high accuracy of the analytical calculation. It should be noted, however, that the mean-field approximation has poor accuracy if an NNCRN is very close to its percolation transition point. This can be confirmed by the fact that the distribution distance $d\left(P_{1}^{\text {ana }}, P_{1}^{\text {num }}\right)$ as a function of the average degree $\langle k\rangle$ displays a sharp peak and takes the maximum value near (or slightly larger than) the critical point $\langle k\rangle_{\mathrm{c}}$ as shown by Table This is because the mean-field treatment ignores the fluctuation of the size of the component containing the source node $j_{k^{\prime}}$, while the component-size distribution is very wide near the percolation threshold.

TABLE I. Maximum values $d_{\max }$ of $d\left(P_{1}^{\text {ana }}, P_{1}^{\text {num }}\right)$ as a function of the average degree for NNCRNs with specific values of $\varrho$ and the same degree distribution as Erdös-Rényi random graphs, the average degree $\langle k\rangle_{\max }$ giving $d_{\max }$, and the critical average degree $\langle k\rangle_{\mathrm{c}}$ for the percolation transition. $\langle k\rangle_{\mathrm{c}}$ 's for $\varrho=0.8$ and $-0,8$ have been estimated numerically by evaluating the largest component size. For $\varrho=0.0,\langle k\rangle_{\mathrm{c}}$ is known to be unity [2].

\begin{tabular}{lccc}
\hline \hline & $\varrho=0.8$ & $\varrho=0.0$ & $\varrho=-0.8$ \\
\hline$d_{\max }$ & 0.66 & 0.66 & 0.57 \\
$\langle k\rangle_{\max }$ & 1.05 & 1.10 & 1.25 \\
$\langle k\rangle_{\mathrm{c}}$ & 0.8 & 1.0 & 1.1 \\
\hline \hline
\end{tabular}



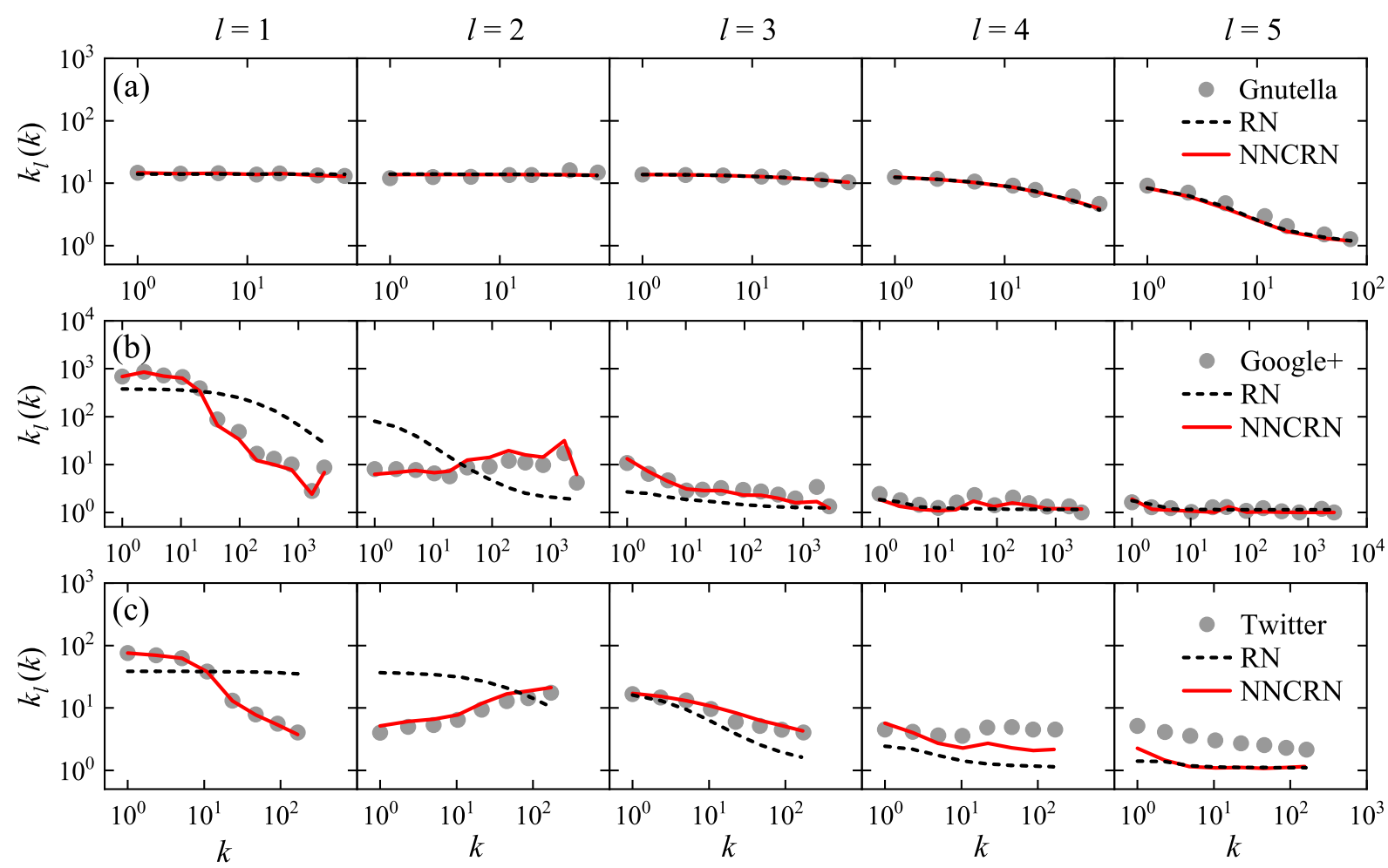

FIG. 4. (Color online) Average degree $k_{l}(k)$ of the $l$ th neighbor nodes of a node of degree $k$ for (a) the Gnutella network, (b) Google+ network, and (c) Twitter network. Gray symbols represent $k_{l}(k)$ for the real-world networks at fixed values of $l$. Dashed black lines indicate $k_{l}(k)$ for the corresponding random networks with the same degree distributions as the real-world networks. Solid red lines are for the NNCRNs with the same NNDCs.

\section{REAL-WORLD NETWORKS}

In this section, we demonstrate how detailed information on LRDC in a given network can be obtained from the probability distributions. For this purpose, we first analyze contrasting three networks; the Gnutella network 24], Google+ network [25], and Twitter network 25]. The Gnutella network $(\langle k\rangle=7.4)$ represents the connection between 10, 876 hosts sharing files by peerto-peer. The Google+ network $(\langle k\rangle=3.3)$ represents the friendship relation between 23,628 users of Google+. The Twitter network $(\langle k\rangle=2.8)$ is comprised of the follower/following relationship between 23, 370 accounts. The average shortest path distances are $\langle l\rangle=4.64$, 4.03, and 6.30 for the Gnutella, Google+, and Twitter networks, respectively. In analyses of these networks, weights and directions of edges are ignored.

Symbols in Fig. 4 shows the $k$ dependence of the average degree of the $l$ th neighbor nodes $k_{l}(k)$ defined by Eq. (2) for these networks. The probability distribution $P\left(k^{\prime} \mid k, l\right)$ is measured directly from the network data. Dashed black lines and red solid lines indicate $k_{l}(k)$ for corresponding random networks with the same degree distributions and NNCRNs with the same NNDCs obtained from $P_{0}\left(k^{\prime} \mid k, l\right)$ and $P_{1}\left(k^{\prime} \mid k, l\right)$, respectively. The probability distributions $P_{0}\left(k^{\prime} \mid k, l\right)$ and $P_{1}\left(k^{\prime} \mid k, l\right)$ are calculated analytically by using $P(k)$ and $P_{\mathrm{nn}}\left(k^{\prime} \mid k\right)$ of the original networks. Gray symbols for the Gnutella network coincide with the dashed black and solid red lines for any $l$ from $l=1$ to 5 , which means that the Gnutella network is totally uncorrelated. In contrast, the results for the Google+ and the Twitter networks deviate from $k_{l}(k)$ for their corresponding random networks (dashed black line) at least for $l \leq 3$. This implies that these networks exhibit LRDCs. The result for the Google+ network agrees well with that for the corresponding NNCRN (solid red line) for any $l$. Therefore, we can conclude that the LRDC in the Google+ network is induced by an NNDC, i.e., the LRDC is extrinsic. On the other hand, $k_{l}(k)$ for the Twitter network differs from both the dashed black and solid red lines for $l \geq 4$, which indicates that the LRDC in the Twitter network is intrinsic. As illustrated by these examples, real-world networks are classified into three types: uncorrelated networks, only nearest-neighbor degree correlated networks (extrinsically long-range correlated networks), 
and intrinsically long-range correlated networks.

We have examined quantitatively LRDCs in many real-world networks by utilizing the distribution distance $d\left(P, P^{\prime}\right)$ defined by Eq. (13). The strength of LRDC in a given network $G$ is quantified by the distance $d\left(P, P_{0}\right)$ between the joint probability distributions $P\left(k, k^{\prime}, l\right)$ for $G$ and $P_{0}\left(k, k^{\prime}, l\right)$ for the corresponding random network with the same degree distribution as $G$. In addition, the strength of the intrinsic character of LRDC in the network $G$ is measured by the distance $d\left(P, P_{1}\right)$ between $P\left(k, k^{\prime}, l\right)$ and $P_{1}\left(k, k^{\prime}, l\right)$ for the NNCRN corresponding to $G$. The quantity $d\left(P, P_{1}\right)$ becomes zero or unity if the LRDC in $G$ is completely extrinsic or intrinsic, respectively. The results for 21 real-world networks are summarized in Table II. Except for the first four networks with low values of $d\left(P, P_{0}\right)$, LRDCs in many real-world networks present a strong intrinsic character with large values of $d\left(P, P_{1}\right)$. It is interesting to notice that networks showing large values of $d\left(P, P_{0}\right)$ give large $d\left(P, P_{1}\right)$ 's. This means that LRDCs in strongly correlated real networks are dominated by intrinsic ones.

It should be noted that three AS-level Internet graphs show strongly disassortative mixing with large negative $\varrho$ while $d\left(P, P_{0}\right)$ is close to zero. An uncorrelated network with $d\left(P, P_{0}\right)=0$ can show disassortative mixing if the degree distribution is highly inhomogeneous, which is known as structural disassortativity [26]. For example, a randomly connected network with the degree distribution $P(k)=\left[\delta_{k, N-1}+(N-1) \delta_{k, 1}\right] / N$ (star-graph degree distribution) has $\varrho=-1$ independently of the network

TABLE II. Characteristics of LRDCs in real-world complex networks. $N,\langle k\rangle$, and $\varrho$ are the network size, average degree, and Spearman's rank correlation coefficients, respectively. The quantities $d_{0}$ and $d_{1}$ represent the values of $d\left(P, P_{0}\right)$ and $d\left(P, P_{1}\right)$, respectively.

\begin{tabular}{|c|c|c|c|c|c|c|}
\hline Network & $N$ & $\langle k\rangle$ & $\varrho$ & $d_{0}$ & $d_{1}$ & Ref. \\
\hline Gnutella & 10,876 & 7.4 & 0.00 & 0.11 & 0.13 & [24] \\
\hline Internet (AS level) (1) & 10,515 & 4.1 & -0.55 & 0.17 & 0.25 & [27] \\
\hline Internet (AS level) (2) & 26,475 & 4.0 & -0.53 & 0.21 & 0.25 & {$[25]$} \\
\hline Internet (AS level) (3) & 22,963 & 4.2 & -0.51 & 0.19 & 0.26 & [28] \\
\hline Google+ & 23,628 & 3.3 & -0.74 & 0.63 & 0.31 & [25] \\
\hline Email & 36,692 & 10.0 & -0.01 & 0.37 & 0.40 & {$[24]$} \\
\hline WWW (1) & 415,624 & 11.4 & -0.32 & 0.87 & 0.41 & {$[25]$} \\
\hline Brightkite & 58,228 & 7.4 & 0.22 & 0.54 & 0.41 & {$[25]$} \\
\hline Facebook & 63,731 & 25.6 & 0.38 & 0.73 & 0.43 & [25] \\
\hline Coauthor (1) & 18,771 & 21.1 & 0.35 & 0.74 & 0.48 & {$[25]$} \\
\hline YouTube & $1,134,890$ & 5.3 & -0.08 & 0.63 & 0.50 & [25] \\
\hline Coauthor (2) & 12,006 & 19.7 & 0.72 & 0.89 & 0.52 & {$[24]$} \\
\hline Actor & 82,583 & 88.8 & 0.34 & 0.73 & 0.57 & 27 \\
\hline Coauthor (3) & 23,133 & 8.1 & 0.26 & 0.71 & 0.61 & $24]$ \\
\hline Twitter & 23,370 & 2.8 & -0.74 & 0.82 & 0.67 & [25] \\
\hline WWW (2) & 325,729 & 6.7 & -0.11 & 0.90 & 0.81 & {$[25]$} \\
\hline Internet (router level) & 192244 & 6.3 & 0.27 & 0.89 & 0.82 & [29] \\
\hline WWW (3) & 255,265 & 15.2 & -0.20 & 0.95 & 0.88 & $25]$ \\
\hline WWW (4) & 685,230 & 19.4 & -0.17 & 0.96 & 0.92 & [25] \\
\hline Protein folding & 132,167 & 3.5 & 0.51 & 0.97 & 0.95 & {$[27]$} \\
\hline Amazon & 334,863 & 5.5 & -0.19 & 0.96 & 0.96 & $25]$ \\
\hline
\end{tabular}

size $N$. In fact, the largest degree node in the network "Internet (AS level) (1)" is connected to more than $20 \%$ of all nodes $\left(N=10,515\right.$ and $\left.k_{\max }=2,277\right)$. For identifying essential degree correlation caused by biased interactions in distinction from apparent structural disassortativity due to inhomogeneous degree distribution, we need to investigate the difference between $P\left(k, k^{\prime}, l\right)$ and $P_{0}\left(k, k^{\prime}, l\right)$ in addition to an assortativity measure like $\varrho$.

In order to understand more deeply how degrees correlate in a network with intrinsic LRDC, we measure the average shortest path distance between two nodes of degrees $k$ and $k^{\prime}$ defined by Eq. (3). It is convenient to introduce the rescaled average shortest path distance $\left\langle l\left(k, k^{\prime}\right)\right\rangle_{\text {res }}$ defined by

$$
\left\langle l\left(k, k^{\prime}\right)\right\rangle_{\mathrm{res}}=\frac{\sum_{l} l \tilde{P}\left(l \mid k, k^{\prime}\right)}{\sum_{l} l \tilde{P}_{1}\left(l \mid k, k^{\prime}\right)},
$$

where the denominator is the average path distance for the corresponding NNCRN. Figure 5 shows the results for three real-world networks with intrinsic LRDCs, the Twitter network [25], protein folding network [27], and WWW in the domain of the University of Notre Dame 25], which are listed in Table II. The results of $\left\langle l\left(k, k^{\prime}\right)\right\rangle_{\text {res }}$ for these networks are, in common, larger than those for the corresponding NNCRNs (flat meshed planes). This implies that the average path distances of the original networks are on average enlarged by their intrinsic LRDCs. Concerning the Twitter network, $\left\langle l\left(k, k^{\prime}\right)\right\rangle_{\text {res }}$ is evenly enlarged almost independently of $k$ and $k^{\prime}$. In contrast, for the protein folding network, the increase of $\left\langle l\left(k, k^{\prime}\right)\right\rangle_{\text {res }}$ for low $k$ and $k^{\prime}$ is much larger than that for high degrees. This implies that there exists long-range repulsive correlation between low degree nodes in the protein folding network. On the other hand, $\left\langle l\left(k, k^{\prime}\right)\right\rangle_{\text {res }}$ for the WWW is notably enlarged at high $k$ and $k^{\prime}$. This shows that the WWW has long-range repulsive correlation between high degree nodes. As demonstrated above, we can obtain detailed information on LRDCs in complex networks by comparing indices defined from the probability distributions with those for corresponding random networks and NNCRNs.

\section{CONCLUSIONS}

We have presented a method to distinguish extrinsic long-range degree correlations (LRDCs) induced by nearest-neighbor degree correlation (NNDC) and intrinsic ones attributed to other factors. In a previous work, it has been shown that the existence of LRDC in a given network $G$ is confirmed by comparing the probability distributions describing the LRDC in $G$ with those for corresponding random networks which have the same number of nodes $N$ and the same degree distribution $P(k)$ as $G$ [15]. Similarly, whether LRDC is extrinsic or intrinsic can be identified by comparing the probability distributions with those for corresponding nearest-neighbor correlated 
(a) Twitter

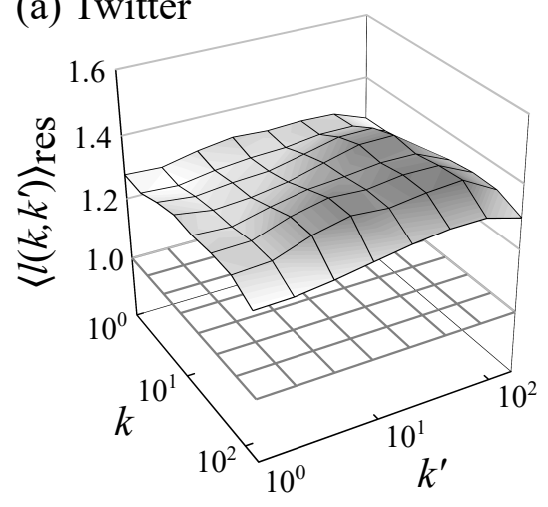

(b) Protein folding

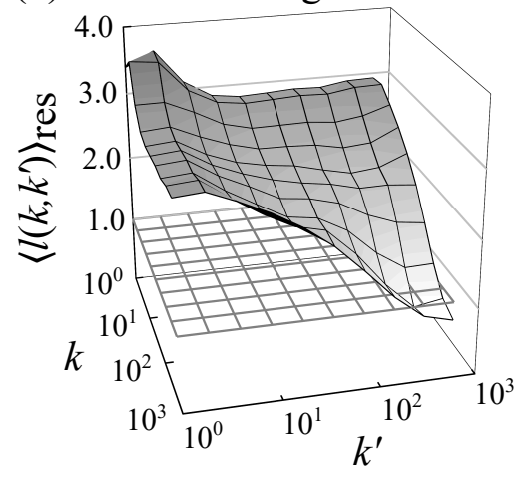

(c) WWW

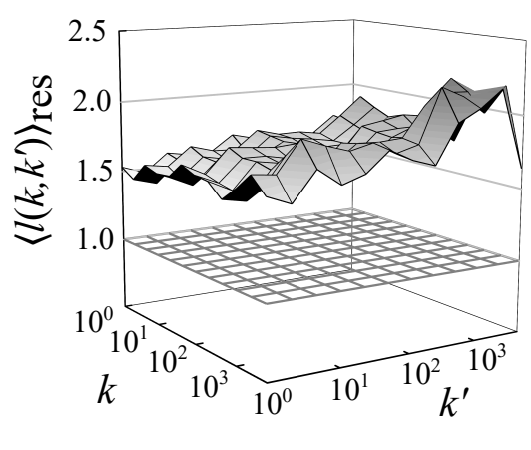

FIG. 5. Rescaled average shortest path distance $\left\langle l\left(k, k^{\prime}\right)\right\rangle_{\text {res }}$ between two nodes of degrees $k$ and $k^{\prime}$ for (a) the Twitter network, (b) protein folding network, and (c) WWW. Two horizontal axes represent $k$ and $k^{\prime}$ in logarithmic scales. The lower flat meshed planes indicate the rescaled average path distances for NNCRNs.

random networks (NNCRNs) which are maximally randomized under the constraint of the same $N$ and NNDC as $G$. We have given a way to calculate the probability distributions for an NNCRN with a specific $N$ and NNDC within the mean-field approximation. The analytical results agree quite well with numerically calculated ones. Finally, we applied our method to several real-world networks and found that most of networks in the real world are intrinsically long-range correlated though there exist uncorrelated and extrinsically long-range correlated networks. Furthermore, we demonstrated that some indices defined from the probability distributions are useful to interpret how degrees correlate in a network with intrinsic LRDC.

It is well known that various properties of complex networks are deeply related to NNDCs. For instance, the robustness [30] and synchronizability of networks are affected by NNDC [31, and fractal scale-free networks show nearest-neighbor disassortative mixing by degree. We need to elucidate how these properties are related to intrinsic LRDCs. In particular, high degree (hub) nodes in fractal scale-free networks are empirically known to repel each other at long distance. Thus, the relation of such a long-range repulsive correlation between hubs to the fractal property must be clarified. Furthermore, it is also quite interesting to study the influence of LRDCs on the percolation problem or random-walk process on a correlated network, because fractal networks are fragile against node elimination and show anomalously slow diffusion. Such studies require efficient indices to extract specific aspects of intrinsic LRDCs. We have employed, in this paper, the average degree of the $l$ th neighbor nodes $k_{l}(k)$ and the rescaled average shortest path distance $\left\langle l\left(k, k^{\prime}\right)\right\rangle_{\text {res }}$ to characterize intrinsic LRDCs. The distribution distance $d\left(P, P^{\prime}\right)$ is also useful to quantify the strength of intrinsic LRDC. One can define many other useful indices from the joint and conditional probability distributions. By utilizing these indices, we can understand precisely the relation between network properties and LRDCs.

\section{ACKNOWLEDGMENTS}

The authors thank S. Mizutaka, T. Hasegawa, and T. Takaguchi for fruitful discussions. This work was supported by a Grant-in-Aid for Scientific Research (No. 16K05466) and a Grant-in-Aid for JSPS Fellows (No. 18J20874) from the Japan Society for the Promotion of Science.
[1] M. Savić, M. Ivanović, L. C. Jain, Complex Networks in Software, Knowledge, and Social Systems (Springer, Berlin, 2019).

[2] R. Albert and A.-L. Barabási, Rev. Mod. Phys. 74, 47 (2002).

[3] M. E. J. Newman, SIAM Rev. 45, 167 (2003).

[4] A.-L. Barabási and R. Albert, Science 286, 509 (1999).

[5] M. E. J. Newman, Phys. Rev. Lett. 89, 208701 (2002).

[6] J. Menche, A. Valleriani, and R. Lipowsky, Phys. Rev. E 81, 046103 (2010).

[7] S.-H. Yook, F. Radicchi, and H. Meyer-Ortmanns, Phys.
Rev. E 72, 045105(R) (2005).

[8] C. M. Schneider, A. A. Moreira, J. S. Andrade Jr., S. Havlin, and H. J. Herrmann, Proc. Natl. Acad. Sci. 108, 3838 (2011).

[9] A.-L. Barabási, Network Science (Cambridge University Press, Cambridge, 2016).

[10] Y. Fujiki, S. Mizutaka, and K. Yakubo, Eur. Phys. J. B 90, 126 (2017).

[11] B. Tadić, S. Thurner, and G. J. Rodgers, Phys. Rev. E 69, 036102 (2004).

[12] M. Boguñá, C. Castellano, and R. Pastor-Satorras, Phys. 
Rev. Lett. 111, 068701 (2013).

[13] R. E. Boulos, A. Arneodo, P. Jensen, and B. Audit, Phys. Rev. Lett. 111, 118102 (2013).

[14] L. W. Swanson, O. Sporns, and J. D. Hahn, Proc. Natl. Acad. Sci. 113, E5972 (2016).

[15] Y. Fujiki, T. Takaguchi, and K. Yakubo, Phys. Rev. E 97, 062308 (2018).

[16] S. Maslov and K. Sneppen, Science 296, 910 (2002).

[17] R. Pastor-Satorras, A. Vázquez, and A. Vespignani, Phys. Rev. Lett. 87, 258701 (2001).

[18] P. Echenique, J. Gómez-Gardeñes, Y. Moreno, and A. Vázquez, Phys. Rev. E 71, 035102(R) (2005).

[19] M. Mayo, A. Abdelzaher, and P. Ghosh, Comput. Soc. Netw. 2, 4 (2015).

[20] A. Arcagni, R. Grassi, S. Stefani, and A. Torriero, Eur. J. Oper. Res. 262, 708 (2017).
[21] S. Melnik and J. P. Gleeson, Preprint arXiv:1604.05521.

[22] N. Litvak and R. van der Hofstad, Phys. Rev. E 87, 022801 (2013).

[23] W.-Y. Zhang, Z.-W. Wei, B.-H. Wang, and X.-P. Han, Physica A 451, 440 (2016).

[24] http://snap.stanford.edu/data/index.html

[25] http://konect.uni-koblenz.de

[26] S. Maslov, K. Sneppen, and A. Zaliznyak, Physica A 333, 529, (2004),

[27] http://cosinproject.eu/data.html

[28] http://www-personal.umich.edu/ mejn/netdata

[29] http://math.bu.edu/people/kolaczyk/datasets.html

[30] A. V. Goltsev, S. N. Dorogovtsev, and J. F. F. Mendes, Phys. Rev. E 78, 051105 (2008).

[31] A. E. Motter, C. S. Zhou, and J. Kurths, Europhys. Lett. 69, 334 (2005). 